\title{
Does early review by a respiratory physician lead to a shorter length of stay for patients with non-severe community-acquired pneumonia?
}

\author{
T Bewick, ${ }^{1}$ V J Cooper, ${ }^{2}$ W S Lim
}

${ }^{1}$ Nottingham City Hospital, Nottingham, UK; ${ }^{2}$ Queens Medical Centre, Nottingham, UK

Correspondence to: Dr T Bewick, Nottingham City Hospital, 55 Stratford Road,

West Bridgford, Nottingham NG2 6AZ, UK; thomasbewick@ doctors.org.uk

Received 22 October 2008 Accepted 27 March 2009

Published Online First

20 April 2009

\begin{abstract}
Background: The aim of this study was to evaluate whether patients with non-severe community-acquired pneumonia (CAP) have a shorter length of stay (LOS) when initially seen by a respiratory physician compared with a non-respiratory physician.
\end{abstract}

Methods: At Nottingham City Hospital, following nurse triage, acute medical patients who are not severely ill are admitted to the consultant-led emergency short stay unit (ESSU). Records of patients seen on ESSU between January 2004 and December 2007 with a clinical discharge code relating to CAP were retrospectively examined. Patients with a diagnosis of cellulitis over the same time period were used as controls. Patients were grouped depending on whether they were seen on their first post-take ward round by a respiratory consultant physician (group A), non-respiratory consultant physician (group B) or on a Saturday or Sunday (group C).

Results: Following exclusions, 426 patients with CAP and 935 patients with cellulitis were analysed. The median LOS for patients with CAP in group A was 1.74 days $(n=123$, interquartile range (IOR) 0.97-4.09) compared with 3.03 days for patients in group $B(n=174$, IQR 1.12-6.23; $p<0.01$ ). There was a larger percentage of discharges within $24 \mathrm{~h}$ of consultant review in group $\mathrm{A}$ (43.1\%) compared with group B $(31.9 \%)$, although this was not statistically significant $(p=0.18)$. There was no statistically significant difference between groups $A$ and $B$ with cellulitis in LOS or percentage discharged within $24 \mathrm{~h}$ of first consultant review.

Conclusion: Patients with non-severe CAP have a shorter hospital LOS when initially seen by a respiratory compared with a non-respiratory physician.

Community-acquired pneumonia (CAP) remains a cause of significant morbidity and mortality in the UK. The annual incidence of CAP admissions to hospitals in England ${ }^{1}$ and elsewhere in the world ${ }^{2}$ has increased significantly in recent years. The cornerstone of management of CAP is accurate severity assessment ${ }^{45}$ which is facilitated by use of a severity assessment tool in conjunction with clinical judgement. Patients with non-severe CAP have a low mortality and therefore may be suitable to be managed at home, or after a short inpatient stay. ${ }^{67}$ Only a small proportion of CAP is managed in hospital, ${ }^{8}$ but this fraction accounts for the majority of the financial burden from this disease. ${ }^{10}$ Outpatient management of non-severe CAP is as safe and effective as hospitalisation, and these patients often prefer outpatient to inpatient treatment. ${ }^{11-14}$
The aim of this study was to investigate whether patients admitted to hospital with nonsevere CAP and managed by respiratory physicians have a shorter length of stay (LOS) compared with patients seen by their general medical colleagues. Our hypothesis was that, compared with general physicians, respiratory specialists are more aware of severity assessment guidelines and have wider experience in managing CAP, and are therefore better able to expedite discharge decisions for patients with non-severe CAP.

\section{METHODS}

\section{Study population}

The study was conducted at Nottingham City Hospital, a busy UK teaching hospital of 1200 beds serving a population of about 700000 people. Admissions with CAP to the medical emergency short stay unit (ESSU) over the 4-year period 20042007 were retrospectively examined. Admissions are streamed to this unit by a nurse triage system that selects patients who are felt to be suitable for early discharge following a short admission. Patients with either a primary or secondary International Classification of Diseases version 10 $(\mathrm{ICD}-10)^{15}$ admission code of pneumonia or lower respiratory tract infection (LRTI) were identified from computer records. Patients admitted over the same time period with an admission code of cellulitis were identified as a control group. The ICD-10 codes used for data retrieval were as follows: J18.0 ("bronchopneumonia"), J18.1 ("lobar pneumonia") J18.9 ("pneumonia, unspecified"), J22 ("unspecified acute lower respiratory tract infection"), L03.0 ("cellulitis of finger and toe"), L03.1 ("cellulitis of other parts of limb") L03.2 ("cellulitis of face"), L03.3 ("cellulitis of trunk") and L03.9 ("cellulitis, unspecified") Information recorded included name, hospital number, date and time of admission, date and time of discharge, readmission within 30 days with the same condition, and all other ICD-10 clinical codes relating to the index admission. All discharge summaries and reports of chest radiographs made by the duty radiologist at the time were available and examined for the CAP cohort. Subjects defined as having CAP included all those with radiological evidence for CAP (as reported by the duty radiologist at the time) and who were managed as CAP during their admission.

Patients from both cohorts were excluded if CAP or cellulitis were not the main reasons for admission, if they were seen by a non-consultant grade doctor and subsequently discharged without 
having seen a consultant physician, or if the patient had an active haematological malignancy requiring admission to a haematology ward. Additionally, patients from the CAP cohort were excluded if they had empyema, hospital-acquired pneumonia, postobstructive pneumonia due to lung cancer, or mesothelioma. Hospital-acquired pneumonia was defined as presentation to hospital within 10 days of a previous admission. In order to confirm that the majority of patients seen on ESSU following nurse triage had non-severe CAP, a random sample of 210 sets of notes representing $50 \%$ of the CAP cohort was examined in more detail. The severity of disease as measured by CURB-65 (new onset confusion, urea $>7 \mathrm{mmol} / 1$, respiratory rate $\geqslant 30$ breaths/min, systolic blood pressure $<90 \mathrm{~mm} \mathrm{Hg}$ and/ or diastolic blood pressure $\leqslant 60 \mathrm{~mm} \mathrm{Hg}$ and age $\geqslant 65$ years $)^{6}$ and modified early warning score (MEWS ${ }^{16}$ was ascertained for these patients. This notes review was also used to validate whether individual patient data had been allocated and analysed correctly according to the criteria described below.

\section{Data analysis}

Consultant-led post-take ward rounds (PTWRs) took place on a daily basis, starting at 9:00 h. Each day the round would be led by a different consultant, determined by a weekly rota, who would review each patient admitted during the preceding $24 \mathrm{~h}$. The consultant rotas for the study period were used to establish whether the lead physician on any particular day was a respiratory specialist or from another medical speciality. Patients were divided up as to whether they saw a consultant respiratory physician (group A), a consultant physician of another speciality (group B) or were first seen by a consultant physician of any speciality on a Saturday or Sunday PTWR (group C). Patients in group C were considered separately, as many of the discharge and diagnostic services at weekends are reduced, potentially prolonging LOS. The primary outcome measure was LOS. Secondary outcome measures were the proportion of patients that were discharged in the $24 \mathrm{~h}$ following first consultant physician review, the proportion of patients that were readmitted within 30 days of the index admission, and 30 day mortality.

\section{Statistical analysis}

Microsoft Excel 2003 (Microsoft Corporation 1985-2003) was used to store and manage the data. Statistical analysis was performed using Statistical Package for the Social Sciences (SPSS) version 16.0 for windows (SPSS 1989-2007). A KruskalWallis test was used to compare non-parametric data on LOS, with the null hypothesis being that early review by a respiratory physician results in no difference in LOS compared with review by a non-respiratory specialist or on a Saturday or Sunday. Pearson $\chi^{2}$ test was used to compare proportions of patients discharged within $24 \mathrm{~h}$ of their first PTWR. A p value of $<0.05$ was taken as statistically significant.

\section{RESULTS}

\section{Patient characteristics}

A total of 1093 patients admitted to ESSU were included in the CAP cohort and 1117 in the cellulitis cohort. The following patients were excluded from the analysis: (1) 595 patients in the CAP cohort who were not treated for CAP or did not have acute pulmonary infiltrates on chest radiography; (2) 46 patients in the CAP cohort and 172 patients in the cellulitis cohort who were discharged without seeing a consultant; and (3) 26 patients in the CAP cohort and 10 patients in the cellulitis cohort who met other exclusion criteria. These data are summarised in fig 1 and table 1 .

Patient characteristics are described in table 2. From the random selection of 210 (50\%) admissions in the CAP cohort examined to establish whether patients had mostly non-severe CAP, $183(87.1 \%)$ had a CURB-65 score of 0 or 1, 18 (8.6\%) had a score of 2 , and $9(4.3 \%)$ had a score of $\geqslant 3$. A total of 178 (84.8\%) had a MEWS score of $<5$. The 30 day mortality within the CAP cohort after exclusions was $5.2 \%$. These data suggest that the study cohort was comprised mainly of patients with non-severe CAP.

No statistically significant difference with regards to age, sex or comorbidity was found between the groups A, B and C in both cohorts. For the patients in whom severity scores were calculated, $88.2,87.1$ and $86.4 \%$ of patients in groups A, B and $\mathrm{C}$, respectively, had a CURB-65 score of 0 or 1 ; a similar proportion of scores was calculated for each of the three groups. Twenty-four (11\%) of the patients within the random selection examined in more detail were found to have been misallocated to groups $\mathrm{A}$ and $\mathrm{B}$ based on computer records, with 11 patients incorrectly allocated to group A and 13 patients incorrectly allocated to group $\mathrm{B}$. The results presented below relate to data analysed following the reallocation of these patients to the correct groups.

\section{Length of stay}

These results are summarised in table 3. Median LOS within the CAP cohort was significantly shorter in group A compared with group B (1.74 days vs 3.03 days; $p<0.01)$. In the cellulitis cohort, there was no statistically significant difference between median LOS (group A 2.86 days vs group B 2.61 days; $p=0.21$ ). Median LOS for admissions at the weekend (group C) was 2.70 days for the CAP cohort and 2.80 days for the cellulitis cohort.

Within the CAP cohort, more patients in group A (43.1\%) were discharged within $24 \mathrm{~h}$ of the first PTWR compared with group B $(31.9 \%)$, but the difference did not achieve significance at the $5 \%$ level $(p=0.18)$. Overall, there were significantly more patients discharged within $24 \mathrm{~h}$ following weekday PTWRs (groups A and B) compared with Saturday and Sunday PTWRs (group C; $38.4 \%$ vs $22.5 \%, p=0.001$ ). In the cellulitis cohort, the proportions discharged within $24 \mathrm{~h}$ were $24.9,31.5$ and $26.3 \%$ for groups A, B and C, respectively. Readmission rates were similar within both cohorts between groups $\mathrm{A}$ and $\mathrm{B}$. However, there was a statistically significant increase in the readmission rate for cellulitis in those first admitted at a weekend compared with those admitted on a weekday (6.5\% vs $3.5 \% ; p=0.04$ ).

\section{DISCUSSION}

This is the first study to our knowledge that compares the front-door consultant-led management of non-severe CAP between respiratory and non-respiratory physicians. Our data suggest that early review by a respiratory physician significantly reduces LOS without affecting the readmission rate. In keeping with the decrease in LOS, there was a higher percentage of discharges within $24 \mathrm{~h}$ of consultant review in group A compared with group B, although this was not statistically significant.

There are several possible explanations for these findings. A previous study ${ }^{17}$ has compared the chance of CAP treatment failure when managed by respiratory versus non-respiratory specialists. Guideline adherence was lower and treatment failure 


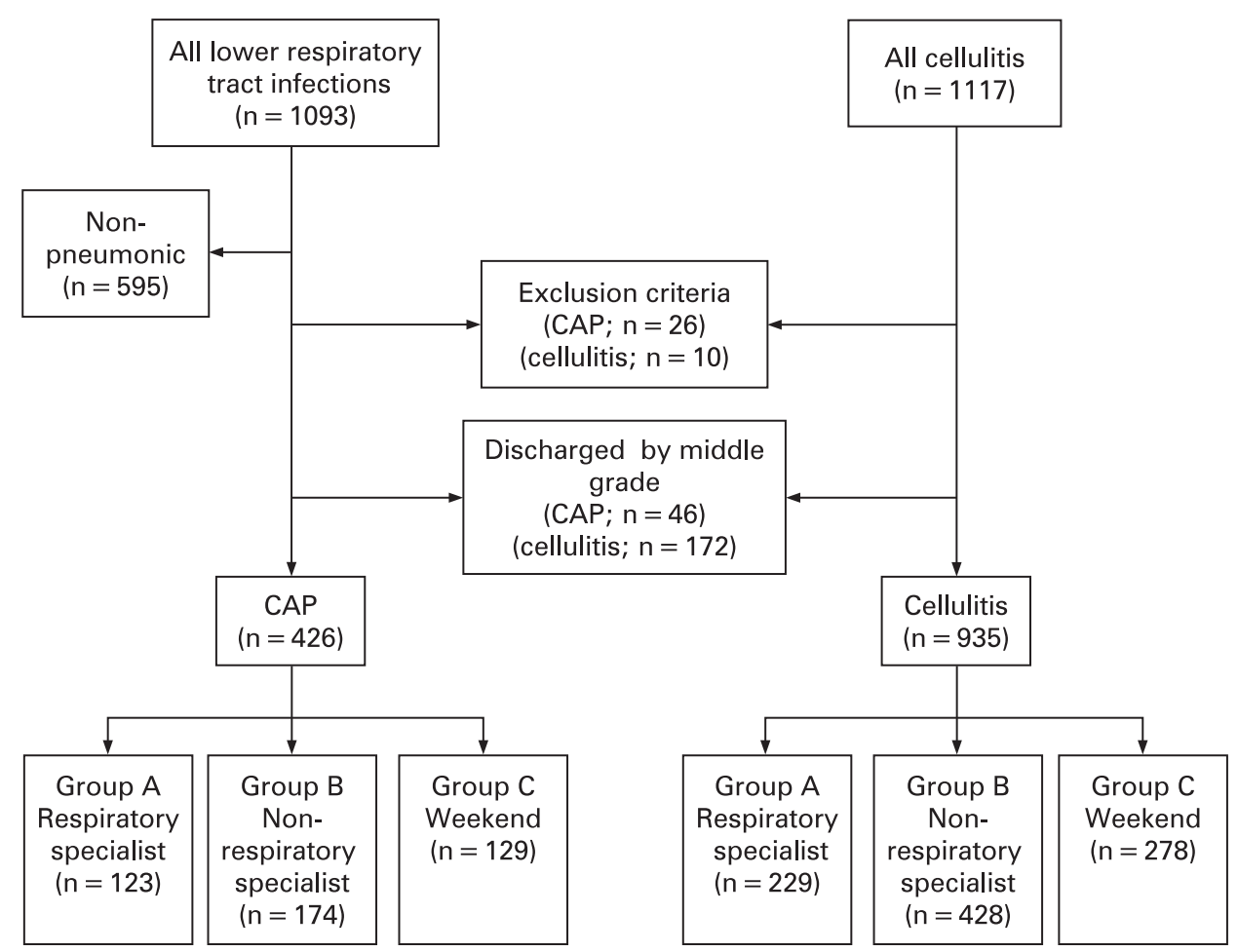

Figure 1 Flow diagram illustrating the distribution of patients included in the study. Group A, first seen by a respiratory physician; group B, first seen by a non-respiratory physician; group $\mathrm{C}$, seen on a weekend post-take ward round. CAP, community-acquired pneumonia.

higher if the patient was managed by a non-respiratory specialist. The study concluded that this difference was due to the better access respiratory specialists had to CAP guidelines, facilitating prompt decision making. The data also suggested that guideline adherence by respiratory specialists promoted a more guideline-based management by non-specialists, implying that respiratory specialists have a training role in the management of CAP. An intensive period of CAP guideline education has been shown significantly to reduce LOS and decrease allcause 30 day mortality in a hospital setting, ${ }^{18}$ suggesting that familiarity with CAP guidelines can have an impact on LOS. The CURB-65 score is the CAP severity assessment tool used within our hospital, and is well known to both junior and senior members of the acute medical team, regardless of their speciality. Nevertheless respiratory physicians may have greater familiarity with these guidelines and their implications. In addition, their more extensive experience in managing CAP may lead to greater confidence in discharging patients earlier.

Patients admitted with cellulitis were selected as a control group because this is a common general medical condition that has no specific affinity to any medical speciality covered by the admitting general medical consultants at this hospital. No difference in the management of cellulitis, and therefore in LOS,

Table 1 Exclusions from the community-acquired pneumonia cohort $(n=1093)$ and the reason for exclusion

\begin{tabular}{lr}
\hline Reason & n \\
\hline No radiographic abnormality consistent with infection & 595 \\
Postobstructive pneumonia & 6 \\
Haematology admission & 7 \\
Hospital-acquired pneumonia & 5 \\
Mesothelioma & 3 \\
Empyema & 5 \\
\hline
\end{tabular}

was expected. The LOS in this control group was similar between respiratory and non-respiratory physicians, suggesting that the difference in LOS in the CAP cohort was not because respiratory physicians generally adopt a more aggressive discharge policy compared with non-respiratory physicians, nor because of more efficient processes of care occurring on the days when respiratory physicians led the PTWR.

Hospitals in the UK and Europe are increasingly incorporating medical ESSUs into their models of acute care. Although there is no recognised standard configuration for these units, the most recent UK recommendations suggest that it be a unit located in close proximity to an acute medical unit, staffed by the same team of doctors and consisting of beds for patients who should complete their inpatient care without transfer to a specialist medical bed. ${ }^{19}$ Our ESSU conforms to this model of acute care.

\section{Study weaknesses}

There are several potential weaknesses with this study. It was a retrospective study, and CAP admissions that were incorrectly coded may have been missed. This was guarded against by reviewing not just the records of patients with a primary and secondary admission code of CAP, but also all other patients with lower respiratory tract infections. A substantial proportion of these subsequently turned out to have had CAP when the discharge summaries and chest radiographs were examined. However, there may have been other patients with CAP who were incorrectly coded in other ways.

The patients with CAP seen by respiratory physicians may have had less severe disease compared with those seen by nonrespiratory physicians. However, CURB-65 scores were similar between the groups in the $50 \%$ of the CAP cohort for whom severity scores were calculated. In addition, there were no statistically significant differences between the two groups in terms of age or number of comorbid illnesses. Practically, there 
Table 2 Characteristics of the study population

\begin{tabular}{llll}
\hline Characteristic & Group A & Group B & Group C \\
\hline CAP cohort & $\mathrm{n}=123$ & $\mathrm{n}=174$ & $\mathrm{n}=129$ \\
Mean age (years) & 52.6 & 52.1 & 53.1 \\
Male (\%) & $56(49)$ & $86(49)$ & $57(44)$ \\
Mean number of comorbid illnesses & 2.9 & 3.2 & 3.4 \\
\%CURB65 0-1 (based on 50\% random sample) & 88.2 & 87.1 & 86.4 \\
Cellulitis cohort & $\mathrm{n}=229$ & $\mathrm{n}=428$ & $\mathrm{n}=278$ \\
Mean age (years) & 54.0 & 56.9 & 56.2 \\
Male (\%) & $141(62)$ & $243(57)$ & $158(57)$ \\
Mean number of comorbid illnesses & 3.2 & 3.1 & 3.2 \\
\hline
\end{tabular}

Group A, first seen by a respiratory physician; group B, first seen by a non-respiratory physician; group C, seen on a post-take ward round on a Saturday or Sunday.

CAP, community-acquired pneumonia; CURB- 65 , new onset confusion, urea $>7 \mathrm{mmol} / \mathrm{l}$, respiratory rate $\geqslant 30 \mathrm{breaths} / \mathrm{min}$, systolic blood pressure $<90 \mathrm{~mm} \mathrm{Hg}$ and/or diastolic blood pressure $\leqslant 60 \mathrm{~mm} \mathrm{Hg}$ and age $\geqslant 65$ years.

was no reason to expect a systemic difference in disease severity between the two groups either.

Within the random patient sample whose notes were examined in greater detail, 24 (11\%) patients had been assigned to the incorrect patient group. This discrepancy was probably due to late changes in the ESSU consultant rota that were not officially documented. It is unknown whether patients in the remaining $50 \%$ of the cohort had a similar misallocation rate. The observed differences between groups A and B were greater when these data were analysed following reallocation of these 24 patients compared with analysis without reallocation. If a similar misallocation rate were assumed in the remaining $50 \%$ of the CAP cohort then the differences would be expected to be further exaggerated and therefore do not detract from the main findings of this study.

\section{Conclusion}

Patients with CAP who are not severely ill have a shorter hospital LOS when initially seen by a respiratory compared witha non-respiratory physician. This may have implications for the acute medical service, implying a benefit of early respiratory review of all CAP admissions.

Table 3 Comparison of length of stay, proportion of patients discharged within $24 \mathrm{~h}$ of first PTWR and readmissions within 30 days with nature of first consultant review

\begin{tabular}{llll}
\hline Characteristic & $\begin{array}{l}\text { Respiratory } \\
\text { group A }\end{array}$ & $\begin{array}{l}\text { Non-specialist } \\
\text { group B }\end{array}$ & $\begin{array}{l}\text { Weekend } \\
\text { group C }\end{array}$ \\
\hline CAP cohort & $\mathrm{n}=123$ & $\mathrm{n}=174$ & $\mathrm{n}=129$ \\
Median LOS in days & $1.74^{*}$ & $3.03^{*}$ & $2.70^{*}$ \\
IQR & $0.97-4.09$ & $1.12-6.23$ & $1.31-5.38$ \\
\% Discharged within 24 h & 43.1 & 31.9 & 22.5 \\
Readmissions within 30 days (\%) & $5(4.1)$ & $7(4.0)$ & $6(4.7)$ \\
Cellulitis cohort & $\mathrm{n}=229$ & $\mathrm{n}=428$ & $\mathrm{n}=278$ \\
Median LOS in days & 2.86 & 2.61 & 2.82 \\
IOR & $1.32-6.16$ & $1.11-6.07$ & $1.20-5.99$ \\
\% Discharged within 24 h & 24.9 & 31.5 & 26.3 \\
Readmissions within 30 days (\%) & $8(3.5)$ & $15(3.5)$ & $18^{* *}(6.5)$ \\
\hline
\end{tabular}

Group $A$, first seen by a respiratory physician; group $B$, first seen by a non-respiratory physician; group $C$, seen on a Saturday or Sunday PTWR.

${ }^{*} \mathrm{p}<0.01$ Kruskal-Wallis test. ${ }^{* *} \mathrm{p}<0.05 \chi^{2}$ test.

CAP, community-acquired pneumonia; LOS, length of stay; IOR, interquartile range; PTWR, post-take ward round.
Acknowledgements: We would like to thank Louise Oxley and the clinical coding department for their efforts in retrieving the data that made this study possible.

Competing interests: None.

\section{REFERENCES}

1. Trotter CL, Stuart JM, George R, et al. Increasing hospital admissions for pneumonia, England (2008). Emerg Infect Dis 2008;14:727-33.

2. Fry AM, Shay DK, Holman RC, et al. Trends in hospitalisation for pneumonia among persons aged 65 years or older in the United States, 1988-2002. JAMA 2005:294:2712-9.

3. Thomsen RW, Riis A, Norgaard M, et al. Rising incidence and persistently high mortality of hospitalized pneumonia: a 10-year population-based study in Denmark. J Intern Med 2006;259:410-7.

4. Macfarlane JT, Boldy D. 2004 update of BTS pneumonia guidelines: what's new? Thorax 2004;59:364-6.

5. Man SY, Lee N, Ip M, et al. Prospective comparison of three predictive rules for assessing severity of community-acquired pneumonia in Hong Kong. Thorax 2007:62:348-53.

6. Lim WS, van der Eerden MM, Laing $R$, et al. Defining community-acquired pneumonia severity on presentation to hospital: an international derivation and validation study. Thorax 2003;58:377-82.

7. Fine MJ, Auble TE, Yealy DM, et al. A prediction rule to identify low risk patients with community-acquired pneumonia. N Engl J Med 1997;336:243-50.

8. Woodhead MA, Macfarlane JT, McCracken JS, et al. Prospective study of the aetiology and outcome of pneumonia in the community. Lancet 1987;329:671-4.

9. Guest JF, Morris A. Community-acquired pneumonia: the annual cost to the National Health Service in the UK. Eur Resp J 1997:10:1530-4.

10. Sun HK, Nicolau DP, Kuti JL. Resource utilisation of adults admitted to a large urban hospital with community-acquired pneumonia caused by streptococcus pneumonia. Chest 2006;130:807-14.

11. Coley CM, Li YH, Medsger AR, et al. Preferences for home vs. hospital care among low-risk patients with community acquired pneumonia. Arch Intern Med 1996;156:1565-71.

12. Carratala J, Fernandez-Sabe N, Ortega L, et al. Outpatient care compared with hospitalization for community-acquired pneumonia: a randomised trial in low-risk patients. Ann Intern Med 2005;142:165-72.

13. Lee RW, Lindstrom ST. Early switch to oral antibiotics and early discharge guidelines in the management of community-acquired pneumonia. Respirology 2007:12:111-6.

14. Labarere J, Stone RA, Obrosky DS, et al. Comparison of outcomes for low-risk outpatients and inpatients with pneumonia: a propensity-adjusted analysis. Chest 2007;131:480-8.

15. World Health Organisation. International classification of diseases and related health problems. 2nd edn. Rome: WHO, 2005.

16. Subbe CP, Kruger $M$, Rutherford $P$, et al. Validation of a modified early warning score in medical admissions. OJM 2001;94:521-6.

17. Menendez R, Torres A, Zalacain R, et al. Guidelines for the treatment of communityacquired pneumonia. Predictors of adherence and outcome. Am J Respir Crit Care Med 2005;172:757-62.

18. Dean NC, Bateman KA, Donnelly SM, et al. Improved clinical outcomes with utilization of a community-acquired pneumonia guideline. Chest 2006;130:794-9.

19. Royal College of Physicians. Acute medical care. The right person in the right setting, first time. Report of the acute medicine task force. London: Royal College of Physicians, 2007. 